\title{
On the Stability of Shield-Driver Circuits
}

\author{
Enrique Mario Spinelli, Senior Member, IEEE, and Ferran Reverter
}

\begin{abstract}
This paper analyzes the stability of shield-driver circuits (SDCs) applied to the measurement of remote signal sources (such as bioelectric signals) and then proposes design ideas to guarantee it. According to the performed analysis, there are at least two factors that explain the potential instability of the SDC: 1) a high order of the SDC transfer function and 2) the parasitic inductance of the interconnecting cable. The former makes the circuit unstable for intermediate values of the output resistance $R_{S}$ of the signal source, whereas the latter makes the circuit unstable for low values of $R_{S}$. These theoretical predictions are experimentally validated using several commercial operational amplifiers.
\end{abstract}

Index Terms-Active shielding, shield-driver circuit (SDC), shielded cable, stability.

\section{INTRODUCTION}

$\mathbf{R}$ EMOTE sensors and signal sources are usually connected to their electronics via shielded cables to reduce the effects of electric field interference. The common practice is to connect the cable shield to the ground potential, which is called passive shielding. However, this configuration is not useful in some applications due to the effects of the parasitic capacitance $C_{\mathrm{SH}}$ of the cable. This capacitance considerably increases the parasitic capacitance to ground, and hence, passive shielding becomes inappropriate for the measurement of grounded capacitive sensors [1], [2] or the characterization of voltmeter input parameters [3]. The capacitance $C_{\mathrm{SH}}$ also decreases the effective input impedance of the readout circuit and can bring about phase and gain errors, which can be a major problem, for example, in impedance measurements [4], [5]. Another effect caused by $C_{\mathrm{SH}}$ is the reduction of the effective common-mode rejection ratio of differential amplifiers, as can happen, for instance, in the measurement of bioelectric signals [6].

The usual way to avoid the previous effects while preserving shielding effectiveness is by applying active shielding, i.e., driving the cable shield at the same potential as that of the inner conductor by using a shield-driver circuit (SDC), as shown in Fig. 1. In this configuration, external interferences are driven to ground through the low output impedance of the SDC, and

Manuscript received October 13, 2008; revised March 19, 2009. First published October 23, 2009; current version published January 7, 2010. This work was supported in part by the Spanish Ministry of Education and Science and the European Regional Development Fund under Project DPI2006-04017 and in part by the Universidad Nacional de La Plata under Project I127. The Associate Editor coordinating the review process for this paper was Dr. Theodore Laopoulos.

E. M. Spinelli is with the Industrial Electronics, Control, and Instrumentation Laboratory (LEICI), Facultad de Ingeniería, Universidad Nacional de La Plata (UNLP)-Consejo de Investigaciones Científicas y Técnicas (CONICET), 1900 La Plata, Argentina (e-mail: spinelli@ing.unlp.edu.ar).

F. Reverter is with the Instrumentation, Sensors and Interfaces Group, Castelldefels School of Technology (EPSC), Technical University of Catalonia (UPC), 08860 Castelldefels, Spain (e-mail: reverter@eel.upc.edu).

Digital Object Identifier 10.1109/TIM.2009.2024698

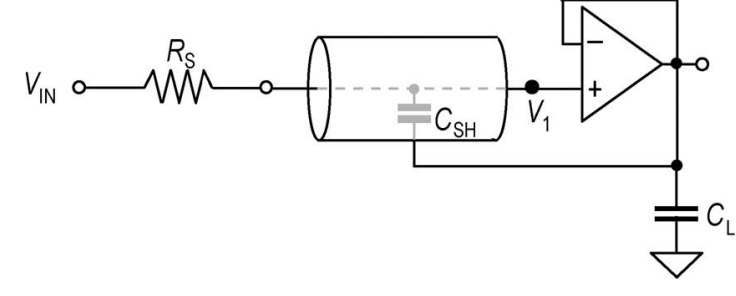

Fig. 1. Measurement of a remote signal source by applying active shielding.

$C_{\mathrm{SH}}$ ideally does not affect the measurement because both cable conductors are at the same potential. However, active shielding has problems in terms of "electronic" instability due to the positive feedback path generated by $C_{\mathrm{SH}}$ [7]. Instability problems are generally solved by means of practical pieces of advice such as decreasing a little bit the gain (e.g., 0.99) of the SDC [5], [6].

To the best of our knowledge, few papers have theoretically analyzed the stability of SDCs. For the measurement of grounded capacitive sensors, stability was carefully analyzed in [8]. For the measurement of bioelectric signals, stability was analyzed in [6]; however, such an analysis used simple circuit models, and no effects of the source impedance on the stability were predicted. This paper improves the approach proposed in [6] and analyzes in detail the stability of SDCs when measuring voltage-modulated signal sources, such as bioelectric signals, or the voltage resulting from impedance measurements.

\section{Circuit Analysis}

Fig. 1 shows an SDC intended for the measurement of a remote signal source, which is modeled by the voltage source $V_{\mathrm{IN}}$ and the resistance $R_{S}$; the output impedance of the signal source is assumed to be only a resistance to simplify the analysis [6]. The SDC is implemented by an operational amplifier (OpAmp) configured as a unity-gain buffer and drives the cable shield to the same potential as that of the inner conductor. The capacitance $C_{\mathrm{SH}}$ is the parasitic capacitance between the inner conductor and the shield of the interconnecting cable; note that a positive feedback path is established through $C_{\mathrm{SH}}$, which could lead to instability problems. The capacitance $C_{L}$ is the capacitive load of the buffer. When a triaxial cable is used and the second shield is connected to ground to be the current return path, $C_{L}$ is almost equal to the parasitic capacitance $C_{\mathrm{SS}}$ between the first and the second shield of the cable.

The circuit in Fig. 1 can be analyzed by the equivalent circuit in Fig. 2, in which $G(s)$ is the transfer function of the buffer when driving the capacitive load $C_{L}$. Let us first assume that $G(s)$ is a first-order low-pass transfer function [6], i.e.,

$$
G(s)=\frac{1}{1+s \tau_{1}}
$$




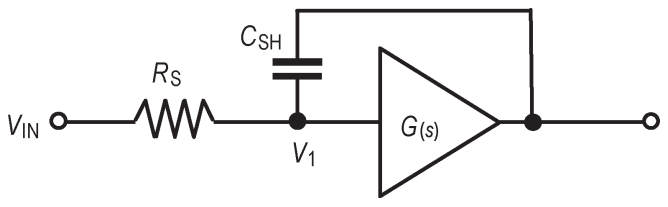

Fig. 2. Equivalent circuit of the circuit shown in Fig. 1.

where $\tau_{1}$ is the time constant of the buffer and is equal to $1 / \omega_{1}$, $\omega_{1}$ being the bandwidth of the buffer in radians per second. Accordingly, the circuit in Fig. 2 has the following transfer function:

$$
T(s)=\frac{V_{1}(s)}{V_{\mathrm{IN}}(s)}=\frac{s+\omega_{1}}{s^{2} \tau_{G}+s+\omega_{1}}
$$

where $\tau_{G}=R_{S} C_{\mathrm{SH}}$. The characteristic polynomial of (2) is of the second order and has positive coefficients. Therefore, its roots have a negative real part [9], and the circuit should be stable for any value of $R_{S}, C_{\mathrm{SH}}$, and $\omega_{1}$ [6].

Practice with the SDC shows instability problems, and this means that the models used before are not good enough to foresee the circuit stability. For this reason, the following sections again analyze the circuit using improved models of both the buffer and the cable. The effects of these improved models are separately evaluated because a simultaneous analysis results in complex analytical expressions that are useless for a conceptual interpretation of the different factors involved. The stability condition resulting from each particular analysis does not completely ensure stability since it results from a noncomplete model of the circuit. The circuit will be stable only when both stability conditions are fulfilled.

\section{A. Transfer Function of the Buffer}

When the buffer drives a high capacitive load $C_{L}$, its transient response significantly differs from the first-order response previously assumed. For this reason, we proceed to analyze the circuit when $G(s)$ is a second-order transfer function, i.e.,

$$
G(s)=\frac{\omega_{2}^{2}}{s^{2}+2 \xi_{2} \omega_{2} s+\omega_{2}^{2}}
$$

where $\omega_{2}$ is the undamped natural frequency, and $\xi_{2}$ is the damping ratio. These parameters depend on the OpAmp features and also on the capacitive load, which is almost equal to the parasitic capacitance $C_{\mathrm{SS}}$ of the cable. Consequently, since $C_{\mathrm{SS}}$ depends on the cable length, then $G(s)$ also depends on the cable length. The effects of the input capacitance $C_{\mathrm{IN}}$ of the OpAmp are also considered here, as shown in Fig. 3.

Using (3), the circuit in Fig. 3 has the transfer function $T(s)$ in (4), shown at the bottom of the page, where $\tau_{i}=R_{S} C_{\mathrm{IN}}$.

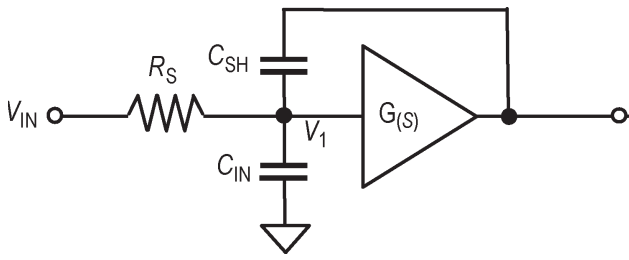

Fig. 3. Equivalent circuit of the circuit shown in Fig. 1 when the input capacitance of the OpAmp is considered.

Considering that $\tau_{i} \ll \tau_{G}$, the characteristic polynomial of (4) can be simplified to

$$
s^{3} \tau_{G}+s^{2}\left(2 \xi_{2} \omega_{2} \tau_{G}+1\right)+s\left(\omega_{2}^{2} \tau_{i}+2 \xi_{2} \omega_{2}\right)+\omega_{2}^{2} .
$$

Applying the Routh-Hurwitz stability criterion [9] in (5) and assuming $C_{\mathrm{IN}} \ll C_{\mathrm{SH}}$, the stability condition is

$$
4 \xi_{2}^{2}+2 \xi_{2}\left(\omega_{2} R_{S} C_{\mathrm{IN}}+\frac{1}{\omega_{2} R_{S} C_{\mathrm{SH}}}\right)>1 .
$$

According to (6), stability depends on several parameters. High values of $\xi_{2}$ and low values of $C_{\mathrm{SH}}$ are recommended to ensure stability. Stability could also be improved by using a high value of $C_{\mathrm{IN}}$, but this is not appropriate because the purpose of the SDC is to keep the input capacitance to ground low. Stability also depends on the values of $R_{S}$ and $\omega_{2}$ so that the circuit is stable for low and high values of $R_{S}$ and $\omega_{2}$, but not for intermediate values. For instance, Fig. 4 shows the function $f$ of $R_{S}$, i.e.,

$$
f\left(R_{S}\right)=4 \xi_{2}^{2}+2 \xi_{2}\left(\omega_{2} R_{S} C_{\mathrm{IN}}+\frac{1}{\omega_{2} R_{S} C_{\mathrm{SH}}}\right)
$$

which points out that the circuit is stable (i.e., $f\left(R_{S}\right)>1$ ) for $R_{S}<R_{S, A}$ and $R_{S}>R_{S, B}$, but not for intermediate values. This dependence on the value of $R_{S}$ can be critical in those applications where $R_{S}$ is not constant, for example, in biopotential measurements [10] and impedance tomography [11].

From (7), we can determine the minimum value of $f$ (i.e., $f_{\min }$ ) and the value of $R_{S}$ (i.e., $R_{S, C}$ ) that brings about $f_{\min }$ (Fig. 4), i.e.,

$$
\begin{aligned}
R_{S, C} & =\frac{1}{\omega_{2} \sqrt{C_{\mathrm{IN}} C_{\mathrm{SH}}}} \\
f_{\text {min }} & =f\left(R_{S, C}\right)=4 \xi_{2}^{2}+4 \xi_{2} \sqrt{C_{\mathrm{IN}} / C_{\mathrm{SH}}} .
\end{aligned}
$$

From (6) and (7), the circuit is clearly stable if $f_{\min }$ is greater than 1 . The parameter $f_{\min }$ depends on the ratio $C_{\mathrm{IN}} / C_{\mathrm{SH}}$, but this is quite variable since $C_{\mathrm{SH}}$ depends on the cable type and length and $C_{\mathrm{IN}}$ depends on the OpAmp used. Therefore, a good option to ensure the stability of the circuit (i.e., $f_{\min }>1$ ) for any ratio $C_{\mathrm{IN}} / C_{\mathrm{SH}}$ is to select a buffer with $4 \xi_{2}^{2}$ higher than 1

$$
T(s)=\frac{V_{1}(s)}{V_{\mathrm{IN}}(s)}=\frac{s^{2}+2 \xi_{2} \omega_{2} s+\omega_{2}^{2}}{s^{3}\left(\tau_{i}+\tau_{G}\right)+s^{2}\left[2 \xi_{2} \omega_{2}\left(\tau_{i}+\tau_{G}\right)+1\right]+s\left(\omega_{2}^{2} \tau_{i}+2 \xi_{2} \omega_{2}\right)+\omega_{2}^{2}}
$$




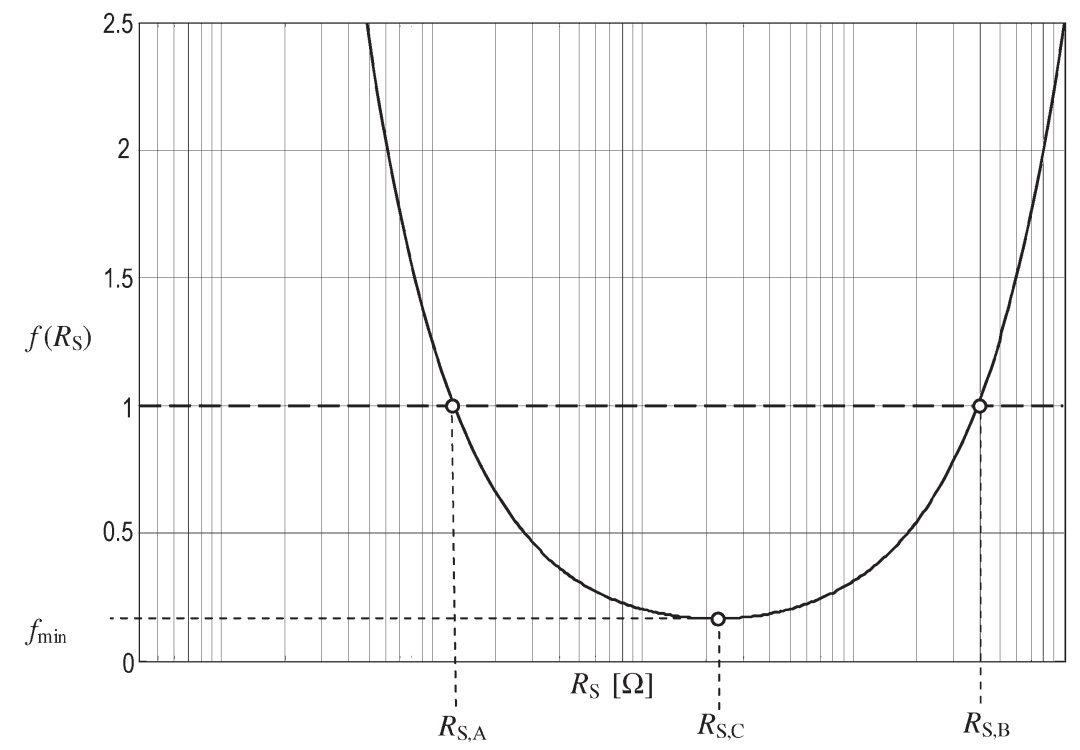

Fig. 4. Typical response of the function $f\left(R_{S}\right)$ described by (7).

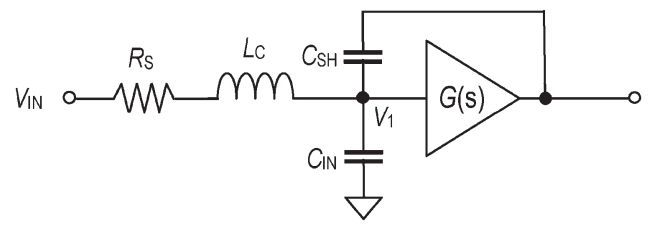

Fig. 5. Equivalent circuit of the circuit shown in Fig. 1 when the cable inductance is considered.

or, in other words, $\xi_{2}$ higher than 0.5 . Note that the buffer must have this damping ratio when driving the capacitive load $C_{L}$. For this reason, it is advisable to select an OpAmp able to drive high-value capacitive loads and/or to apply appropriate compensation methods, for example, adding a resistor (either out or in the loop) between the OpAmp output and the capacitive load [12].

Once the stability of the SDC is guaranteed, the next issue to analyze is the transient response of the voltage at node $V_{1}$. Although this analysis is not the main objective of this paper, next, we provide some comments about it. The transient response can be found from (4), but there are not simple relationships between the parameters of the circuit and the features of the transient response. However, according to simulations, the step transient response is underdamped when $R_{S}$ is close to the critical values $R_{S, A}$ and $R_{S, B}$ (Fig. 4), whereas it becomes more damped as $R_{S}$ decreases with respect to $R_{S, A}$ or increases with respect to $R_{S, B}$. In addition, the higher the value of $R_{S}$, the slower the transient response.

\section{B. Cable Inductance}

In the previous analyses (Figs. 2 and 3), the interconnecting cable was modeled only with a lumped capacitor $C_{\mathrm{SH}}$. This electric model, however, is not accurate enough for stability analysis, particularly when long cables are used. Such a model can be improved by adding the inductance $L_{C}$ of the current loop between the signal source and the readout circuit, as shown in Fig. 5. A lumped model is considered for the inductance to simplify the analysis and because it was already used and experimentally verified in [8].

The following analysis takes into account the effects of $L_{C}$ but assumes a first-order $G(s)$ to keep a low-complexity model, as explained at the beginning of Section II. Then, assuming (1) and some practical relations between parameters (e.g., $\tau_{i} \ll \tau_{G}$ and $C_{\mathrm{IN}} \ll C_{\mathrm{SH}}$ ), the transfer function of the circuit in Fig. 5 can be approximated to

$$
T(s)=\frac{V_{1}(s)}{V_{\mathrm{IN}}(s)} \approx \frac{s+\omega_{1}}{s^{3} L_{C} C_{\mathrm{SH}}+s^{2} \tau_{G}+s\left(\omega_{1} \tau_{i}+1\right)+\omega_{1}} .
$$

Applying the Routh-Hurwitz stability criterion [9] to the characteristic polynomial of (10), the stability condition is

$$
\frac{R_{S}}{\omega_{1}}+R_{S}^{2} C_{\mathrm{IN}}>L_{C}
$$

which shows that high values of $R_{S}$ are appropriate to guarantee stability. For low values of $R_{S}$ and typical values for the rest of parameters, the first term on the left side of (11) predominates over the second term, and then, the stability condition can be simplified to

$$
R_{S}>\omega_{1} L_{C}
$$

According to (12), the circuit becomes unstable for low values of $R_{S}$, particularly when high-bandwidth OpAmps (i.e., a large $\omega_{1}$ ) and long cables (i.e., a large $L_{C}$ ) are used. For example, for $\omega_{1}=2 \pi \cdot 20 \mathrm{MHz}$ and $L_{C}=2 \mu \mathrm{H}$, the stability condition is $R_{S}>251 \Omega$. Therefore, high-bandwidth OpAmps are not advisable when using long interconnecting cables.

\section{EXPERIMENTAL RESULTS AND DISCUSSION}

The stability models developed in Section II were validated by means of the experimental setup shown in Fig. 6. A battery of $1.5 \mathrm{~V}$ was used as a signal source; this battery also provided 


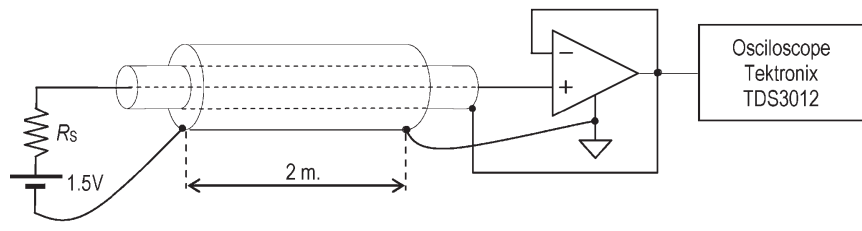

Fig. 6. Experimental setup to validate the stability models developed in Section II.

TABLE I

EXPERIMENTAL VAlues OF THE PARAMETERS $C_{\mathrm{IN}}, \omega_{2}$, AND $\xi_{2}$ OF THE UNITY-GAIN BUFFER WHEN DRIVING A CAPACITIVE LOAD $C_{\mathrm{SS}}=720 \mathrm{pF}$

\begin{tabular}{|c|c|c|c|c|}
\hline & LM358 & TLC2202 & OPA350 & OP07 \\
\hline$C_{\mathrm{IN}}(\mathrm{pF})$ & 3 & 22 & 7 & 7 \\
\hline$\omega_{2}(\mathrm{rad} / \mathrm{s})$ & $2 \pi \cdot 480 \mathrm{k}$ & $2 \pi \cdot 900 \mathrm{k}$ & $2 \pi \cdot 14 \mathrm{M}$ & $2 \pi \cdot 800 \mathrm{k}$ \\
\hline$\xi_{2}$ & 0.08 & 0.15 & 0.3 & 0.55 \\
\hline
\end{tabular}

a dc level to ensure a linear operation for the single-supply OpAmps. A resistor $R_{S}$ (ranging from $10 \Omega$ to $1 \mathrm{M} \Omega$ ) emulated the output resistance of the signal source. Then, the signal was connected to the circuit through a $2-\mathrm{m}$ triaxial cable. This cable was characterized by an impedance analyzer (HP4262A), and the results were $C_{\mathrm{SH}}=192 \mathrm{pF}, C_{\mathrm{SS}}=720 \mathrm{pF}$, and $L_{C}=$ $0.8 \mu \mathrm{H}$. The first shield of the cable was driven by a unity-gain buffer implemented by different commercial OpAmps (LM358, TLC2202, OPA350, and OP07). The second shield was connected to ground, and hence, the capacitive load $C_{L}$ of the buffer was almost equal to $C_{\mathrm{SS}}$. The OpAmps operated at a single supply voltage $(5 \mathrm{~V})$ except for OP07, which operated at $\pm 15 \mathrm{~V}$. The output voltage of the buffer was connected to a digital oscilloscope (Tektronix TDS3012) to display the state (i.e., stable or unstable) of the circuit.

Table I summarizes the experimental values of $C_{\mathrm{IN}}, \omega_{2}$, and $\xi_{2}$ for each tested OpAmp. The parameters $\omega_{2}$ and $\xi_{2}$ were estimated by applying a step of $10 \mathrm{mV}$ at the input of the unity-gain buffer and approximating a second-order response to the output transient response when driving a capacitive load of $720 \mathrm{pF}$. Using the values in Table I and (7), we calculated the theoretical functions $f\left(R_{S}\right)$ for the four OpAmps, which are represented in Fig. 7. According to Fig. 7, the circuit should be unstable (i.e., $f\left(R_{S}\right)<1$ ) for the following intermediate values of $R_{S}$ : between $280 \Omega$ and $670 \mathrm{k} \Omega$ for LM358, between $300 \Omega$ and $24 \mathrm{k} \Omega$ for TLC2202, and between 60 and $1700 \Omega$ for OPA350; OP07 should be always stable since $\xi_{2}>0.5$.

Using (12), we calculated the theoretical minimum value of $R_{S}\left(R_{S, \text { min }}\right)$ to guarantee stability in terms of cable inductance effects. The value of $\omega_{1}$ considered to calculate $R_{S, \min }$ was the experimental value of $\omega_{2}$ shown in Table I; it is true that $\omega_{2}$ corresponds to a second-order response, but it is the best estimation of the buffer bandwidth that we have, particularly when driving a high-value capacitive load. For LM358, TLC2202, and OP07, $R_{S, \text { min }}$ was lower than $5 \Omega$, which means that cable inductance effects are almost negligible in terms of stability. However, for OPA350, $R_{S, \min }=70 \Omega$, and therefore, if we

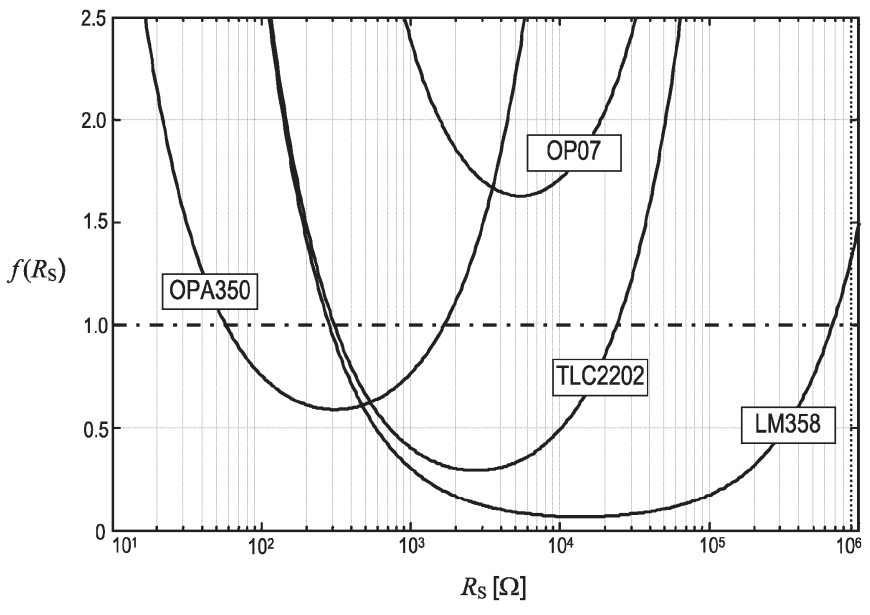

Fig. 7. Function $f\left(R_{S}\right)$ described by (7) for the tested OpAmps.

TABLE II

EXPERIMENTAL RESUltS OF THE STABILITY TESTS FOR DIFFERENT VALUES OF $R_{S}$

\begin{tabular}{|c|c|c|c|c|}
\hline$R_{\mathrm{S}}(\Omega)$ & LM358 & TLC2202 & OPA350 & OP07 \\
\hline 10 & Stable & Stable & Unstable & Stable \\
\hline 50 & Stable & Stable & Unstable & Stable \\
\hline 100 & Stable & Stable & Unstable & Stable \\
\hline $1 \mathrm{k}$ & Unstable & Unstable & Unstable & Stable \\
\hline $10 \mathrm{k}$ & Unstable & Unstable & Stable & Stable \\
\hline $100 \mathrm{k}$ & Unstable & Stable & Stable & Stable \\
\hline $1 \mathrm{M}$ & Stable & Stable & Stable & Stable \\
\hline
\end{tabular}

take into account both stability conditions, the circuit should be unstable for $R_{S}<1700 \Omega$.

Table II shows the experimental results of the stability tests for the different values of $R_{S}$ and for each tested OpAmp. LM358 and TLC2202 were unstable only for intermediate values of $R_{S}$, as predicted in the last paragraph. OPA350 was unstable for low and intermediate values of $R_{S}$, which also agrees with the theoretical predictions. Finally, OP07 was always stable for the values of tested $R_{S}$, which was also predicted before. Therefore, the fact of considering a secondorder response for the buffer and the cable inductance seem to be key points to foresee the circuit stability.

\section{CONCLUSION}

Stability of SDCs depends on the OpAmp's response working as a unity-gain buffer, the output resistance $R_{S}$ of the signal source, and the cable parameters. Due to the high order of the transfer function of the buffer, the circuit becomes unstable for intermediate values of $R_{S}$. A unity-gain buffer with a damping ratio greater than 0.5 avoids such instability problems. On the other hand, due to the parasitic inductance of the interconnecting cable, the circuit becomes unstable for low values of $R_{S}$. To prevent such effects, it is advisable not 
to select a high-bandwidth OpAmp when using long cables. All these theoretical stability conditions have experimentally been verified for different commercial OpAmps.

\section{ACKNOWLEDGMENT}

The authors would like to thank F. López and S. Rodriguez for the technical support.

\section{REFERENCES}

[1] S. M. Huang, A. L. Stott, R. G. Green, and M. S. Beck, "Electronic transducers for industrial measurement of low value capacitances," $J$. Phys. E, Sci. Instrum., vol. 21, no. 3, pp. 242-250, Mar. 1988.

[2] D. Marioli, E. Sardini, and A. Taroni, "High-accuracy measurement techniques for capacitance transducers," Meas. Sci. Technol., vol. 4, no. 3, pp. 337-343, Mar. 1993

[3] I. Lenicek, D. Ilic, and R. Malaric, "Determination of high-resolution digital voltmeter input parameters," IEEE Trans. Instrum. Meas., vol. 57, no. 8, pp. 1685-1688, Aug. 2008.

[4] B. P. Iliev, G. A. M. Pop, and G. C. M. Meijer, "In-vivo blood characterization system," in Proc. IEEE IMTC, Apr. 2006, pp. 1781-1785.

[5] H. G. Goovaerts, T. J. C. Faes, E. Raaijmakers, and R. M. Heethaar, "Some design concepts for electrical impedance measurement," Ann. N.Y. Acad. Sci., vol. 873, no. 1, pp. 388-395, Apr. 1999.

[6] A. C. Meeting van Rijn, A. Peper, and C. A. Grimbergen, "High-quality recording of bioelectric events. Part 1: Interference reduction, theory and practice," Med. Biol. Eng. Comput., vol. 28, no. 5, pp. 389-397, Sep. 1990.

[7] R. Morrison, Solving Interference Problems in Electronics. New York: Wiley, 1995.

[8] F. Reverter, X. Li, and G. C. M. Meijer, "Stability and accuracy of active shielding for grounded capacitive sensors," Meas. Sci. Technol., vol. 17, no. 11, pp. 2884-2890, Nov. 2006.

[9] R. C. Dorf and R. H. Bishop, Modern Control Systems. Upper Saddle River, NJ: Prentice-Hall, 2005.

[10] J. Rosell, J. Colominas, P. Riu, R. Pallàs-Areny, and J. G. Webster, "Skin impedance from $1 \mathrm{~Hz}$ to $1 \mathrm{MHz}$," IEEE Trans. Biomed. Eng., vol. 35, no. 8, pp. 649-651, Aug. 1988.
[11] D. S. Holder, Electrical Impedance Tomography: Methods, History and Applications. Bristol, U.K.: CRC Press, 2005.

[12] Operational amplifier stability compensation methods for capacitive loading applied to TS507, STMicroelectronics, Geneva, Switzerland, 2007. [Online]. Available: http://www.st.com

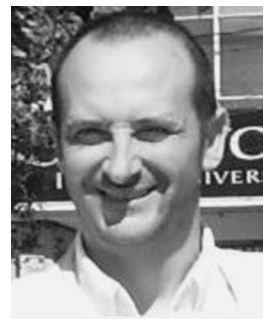

Enrique Mario Spinelli (S'98-M'02-SM'08) was born in Balcarce, Argentina, in 1964. He received the Engineer degree in electronics and the M.S. and $\mathrm{Ph} . D$. degrees from the Universidad Nacional de La Plata (UNLP), La Plata, Argentina, in 1989, 2000, and 2005, respectively.

Since 1990, he has been with the Industrial Electronics, Control, and Instrumentation Laboratory (LEICI), Facultad de Ingeniería, UNLP, working on scientific instrumentation. He is currently a Professor of control systems with the Facultad de Ingeniería, UNLP, and a Researcher with the Consejo Nacional de Investigaciones Científicas y Técnicas (CONICET). His current research interests are analog signal processing and brain control interfaces.

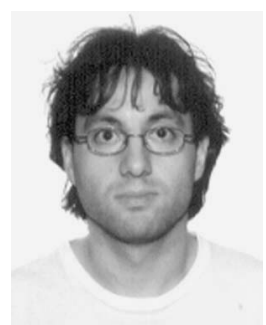

Ferran Reverter was born in Llagostera, Spain, on January 4,1976 . He received the B.Sc. degree in industrial electronic engineering from the University of Girona, Girona, Spain, in 1998, the M.Sc. degree in electronic engineering from the University of Barcelona, Barcelona, Spain, in 2001, and the Ph.D. degree in electronic engineering from the Technical University of Catalonia (UPC), Barcelona, in 2004.

Since 2001, he has been an Assistant Professor of analogue electronics and digital systems with the UPC. From 2005 to 2007, he was a Visiting Postdoctoral Fellow with Delft University of Technology, Delft, The Netherlands. He is the coauthor (with R. Pallàs-Areny) of the book Direct Sensor-to-Microcontroller Interface Circuits (Barcelona: Marcombo, 2005). His research interests are in the field of electronic instrumentation, particularly the design and characterization of interface circuits for sensors. 